\title{
La rehabilitación de la Ciudad de los Ángeles de Secundino Zuazo y la gestión vecinal en la recuperación del patrimonio
}

Francisco Javier González González, Miguel Lasso de la Vega | Universidad

Europea

Daniel Morcillo Álvarez | Universidad Politécnica de Madrid

URL de la contribución <www.iaph.es/revistaph/index.php/revistaph/article/view/4945>

\section{RESUMEN}

La rehabilitación de Ciudad de los Ángeles (Madrid), de Secundino Zuazo, ha supuesto toda una serie de medidas encaminadas a mejorar la habitabilidad de los edificios residenciales, al tiempo que se favorecía el entorno urbano en aspectos tan relevantes como la accesibilidad y la calidad ambiental. El proceso ha sido desarrollado por las administraciones públicas en coordinación y con la participación del movimiento vecinal, de tal manera que los valores patrimoniales existentes en el barrio han sido preservados a través de una suerte de protección ambiental. Este artículo trata de hacer un recorrido por el proceso de rehabilitación seguido, presentando los valores patrimoniales existentes en el barrio, y considerando el papel del "apego al lugar" como medio de protección indirecta del entorno.

\section{Palabras clave}

Ciudad de los Ángeles (Madrid) | Edificios residenciales | Gestión vecinal | Movimiento Moderno | Participación ciudadana | Periferia urbana | Regeneración urbana | Rehabilitación energética | Secundino Zuazo | 
With the

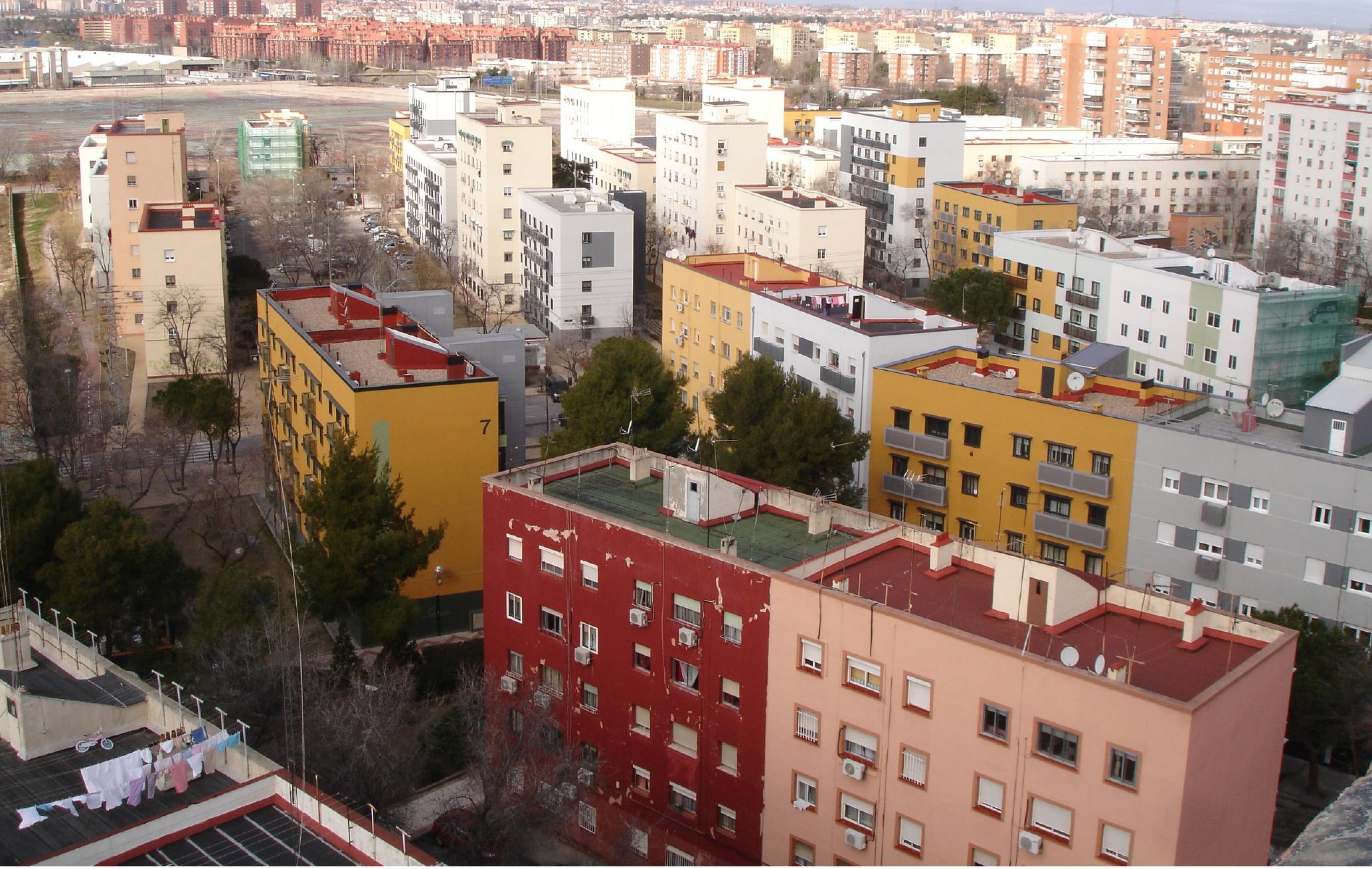

Vista general de Ciudad de los Ángeles | foto el material gráfico que ilustra este artículo pertenece a los autores 


\section{INTRODUCCIÓN. ORÍGENES DE LA REHABILITACIÓN DE CIUDAD DE LOS ÁNGELES}

La rehabilitación de barrios de la periferia contemporánea de nuestras ciudades es una práctica que se ha centrado en la mejora de las condiciones ambientales y de funcionalidad, tanto de edificios como de espacios públicos. Sin embargo, los valores patrimoniales se han quedado en un segundo plano, a pesar de que algunos de estos barrios son ejemplos de cómo el Movimiento Moderno se despliega en el espacio residencial de la ciudad. El caso de la rehabilitación de Ciudad de los Ángeles ha sido uno de los últimos barrios con valores patrimoniales reconocidos que se han mejorado en Madrid, y donde la implicación vecinal ha tenido un papel central en el proceso. Pese a que la preservación patrimonial no ha sido uno de los objetivos de la rehabilitación, el trabajo llevado a cabo ha sido discutido por los agentes implicados de tal manera que ha sido posible compatibilizar una suerte de protección ambiental con las necesidades de mejora de la habitabilidad.

El caso de Ciudad de los Ángeles es significativo en muchos aspectos, pero en lo que nos interesa, cabe resaltar que todo el proceso fuera gestionado por la Empresa Municipal de la Vivienda y Suelo de Madrid (EMVS) y consensuado permanentemente con el movimiento vecinal, a través de la Asociación de Vecinos de la Ciudad de los Ángeles (ASVEYCO). Este hecho hizo que se establecieran mecanismos de participación y control de las decisiones, lo que posibilitó la unidad de criterios y la coherencia de la intervención dentro de la lógica espacial del barrio y sus habitantes.

Localizado en el distrito de Villaverde, al sur de la ciudad de Madrid, el barrio se construye a partir de los años 1960 bajo la influencia de los industriales del entorno y desarrollado en dirección norte sur lindando con la Avenida de Andalucía. Este único punto de conexión con la ciudad y la vinculación con la industria limítrofe han determinado en gran medida el devenir del barrio y la creación de una fuerte identidad vecinal. El carácter del barrio, las homogéneas condiciones de la población y sus problemas cotidianos, laborales y sociales, así como una necesaria apropiación del espacio ante la dejación de funciones de la administración durante décadas, han permitido la construcción de una identidad muy marcada y un "apego al lugar" como respuesta colectiva a los problemas suscitados por el contexto laboral y social.

En lo relativo a la producción del espacio físico, a partir de un trazado original del estudio de Secundino Zuazo, se construye en varias fases hasta los años 1990, manteniendo, pese a ello, un carácter unitario y coherente con la estructura original. Sin embargo, el tipo de promoción y el destinatario final de las viviendas supusieron la elección de sistemas constructivos y materiales que han favorecido la aparición de daños. Las deficientes formas de 
conservación y mantenimiento que se llevaron a cabo hicieron que en los primeros años del siglo $X X$ se iniciara un proceso de movilización para la rehabilitación por parte de ASVEYCO, que terminaría convirtiéndose en su verdadero promotor e impulsor.

Así, en el año 2005 se produce la declaración del Área de Rehabilitación Integral Ciudad de Los Ángeles por Orden de la Consejería de Medio Ambiente y Ordenación de Territorio de la Comunidad de Madrid, iniciándose el proceso de rehabilitación que ha perdurado hasta el año 2018.

El área ocupa una superficie de 59,6 ha, con un total de 7.996 viviendas distribuidas en 441 bloques de los 486 que componen el área y una población estimada actual de 31.465 habitantes a fecha de la declaración del área.

La rehabilitación comprendió tanto el espacio edificado como el espacio libre público. En cuanto a la edificación, se priorizó la estabilidad estructural de los edificios, la accesibilidad y la mejora del comportamiento higrotérmico. Por otra parte, y de manera complementaria, se trazaron los objetivos sobre el entorno urbano, con la idea de eliminar las barreras arquitectónicas existentes, mejorar el medioambiente urbano y aumentar la calidad peatonal de un espacio con una sucesión de espacios libres muy compleja y generosa.

Durante este proceso se ha intervenido sobre 110 edificios, rehabilitando un total de 1.431 viviendas y 29 locales comerciales asociadas a estas. La inversión total ha consistido en 27,15 millones de euros en la rehabilitación de edificios y cerca de 16,5 millones de euros para la mejora de las infraestructuras urbanas. En todo momento se han mantenido como invariables el protagonismo y liderazgo vecinal en el proceso llevado a cabo, aspectos que son necesarios evaluar dentro de un contexto más general.

\section{SECUNDINO ZUAZO EN LA CONSTRUCCIÓN DE LA PERIFERIA MADRILEÑA: VALORES PATRIMONIALES LIGADOS A LA PRODUCCIÓN DEL ESPACIO EN CIUDAD DE LOS ÁNGELES}

A partir de 1950, la Administración Pública y su Instituto Nacional de Vivienda (INV) se convirtieron en el indudable motor de los nuevos barrios, donde se experimentó con materiales constructivos nuevos, soluciones estructurales y distribuciones de carácter mínimo, recogiendo la herencia racionalista de la República y la influencia de las actuaciones extranjeras contemporáneas, a través de las publicaciones y los viajes.

Sin embargo, los sucesivos decretos de bonificación y exención fiscal en la construcción de viviendas sociales no lograban animar al capital privado en 
Sobre la impronta del arquitecto José Fonseca en el INV, concretamente en su Sección de Arquitectura y en las ordenanzas técnicas que desde ella se elaboraron, ver Lasso de la Vega (2008).

\section{2}

Su construcción se ciñe al modelo de promoción inmobiliaria que permitió a gran parte de la población trabajadora acceder a la propiedad de su vivienda. Este paradigma se consolidaría en las décadas posteriores hasta constituir lo que Fernando Roch (2008) denominó como el modelo de propiedad universal. Por otro lado, no hay que descartar que el apoyo de los sectores industriales condicionase el diseño del barrio, apuntalado por la vasta experiencia del estudio Zuazo, y que se plasmó en un diseño urbano en donde el bloque lineal exento, de formas puras, repetitivas y con referencias industriales, se superponen con galerías comerciales de enorme plasticidad gracias a las ligeras marquesinas de hormigón sostenidas por esbeltos pilares circulares de hormigón.

3

A falta de un estudio sobre la figura del arquitecto Manuel Muñoz Monasterio, los arquitectos Carlos Ripoll y Virginia Ripoll señalan: "Muñoz Monasterio no fue un arquitecto representativo de ningún movimiento, ni promotor de ninguna corriente, desarrolló una arquitectura racionalista, sobre todo en su prime ra época de arquitecto, en los años treinta, aunque su salto cualitativo se produce en los cincuenta" (Ripoll Gómez y Ripoll Tolosana 2014).

4

Biblioteca Nacional de España, Fondo Secundino Zuazo, DIBZ/187.

5

En el Archivo de Visado del COAM se registra también la intervención de Secundino Zuazo en la ampliación del barrio en 1968 (Gutiérrez Marcos y Da Rocha 2007). esta empresa, siendo una necesidad del Estado para resolver el que era uno de sus más graves y acuciantes problemas tras la Guerra Civil, el déficit de hogares dignos para una población que había abandonado el medio rural en busca de mejores oportunidades en la capital.

Tras fallidos intentos, la Ley del 15 de julio de 1954 sobre Protección de Viviendas de Renta Limitada, que canceló los regímenes de viviendas protegidas y bonificables, amplió la movilización del crédito financiero, facilitando la participación de los particulares (Ley 84/1961) en la tarea de reconstrucción y desarrollo del parque residencial madrileño. Las reticencias se habían basado en someterse a la férrea supervisión y control del INV, interesado, con José Fonseca al frente de su Sección de Arquitectura ${ }^{1}$, en mantener unos estándares de calidad y funcionalidad que por el contrario mermaban el beneficio lucrativo. Sin embargo, entre las promociones privadas que, en el Madrid de mitad de siglo, se acogen al nuevo Plan Nacional de Viviendas de Renta Limitada (Decreto-ley de 1 de julio de 1955), la Ciudad de los Ángeles merece un lugar destacado.

Su éxito fue reconocido tempranamente por el Estado, al concedérsele la medalla de colaborador del Plan de Urgencia Social de Madrid (Elvira Peña 2003) (Decreto-ley del 13 de noviembre de 1957) a este barrio de la Carretera de Andalucía, surgido a partir de 1951 (Río Lafuente 1984) para alojar a los trabajadores de las industrias próximas, especialmente de la Fábrica Barreiros ${ }^{2}$. En este logro contribuyeron distintos factores, sintetizados en la apuesta de sus arquitectos proyectistas por convertirse en constructores, vitalizando el proceso, y la notable capacidad de aquéllos; la de Javier de Zuazo Bengoa, unida a la experiencia de quienes le acompañaron en esta aventura, Manuel Muñoz Monasterio ${ }^{3} y$, especialmente, su padre, Secundino de Zuazo Ugalde.

Y es que Zuazo es, sin duda, el referente en esta empresa urbana y constructiva, para la que fundó, aunque no participó como socio, la empresa VIRELSA (Viviendas de Renta Limitada SA), poniéndola a nombre de su hijo y sus dos yernos. Quizás lo hizo así porque ya formaba parte de otras tres promotoras: Ia Inmobiliaria de Fomento del Hogar, la Sociedad Anónima Madrileña de Gestión Inmobiliaria y una más "con Federico Turell para conservar y reparar edificios" (Sambricio 1999).

Ese papel secundario en la actividad promotora hizo que también se le supusiera en el proyecto y la construcción de la Ciudad de los Ángeles, pero basta comprobar los muchos documentos firmados de su mano entre los años 1956 y 1961, y que se conservan en su archivo, dibujos y planos de ordenación, manzanas, bloques y torres ${ }^{4}$, con tipos y categorías de viviendas, escuela, mercado y locales comerciales, en la primera, segunda y tercera fases, para comprobar su decisiva implicación ${ }^{5}$. 


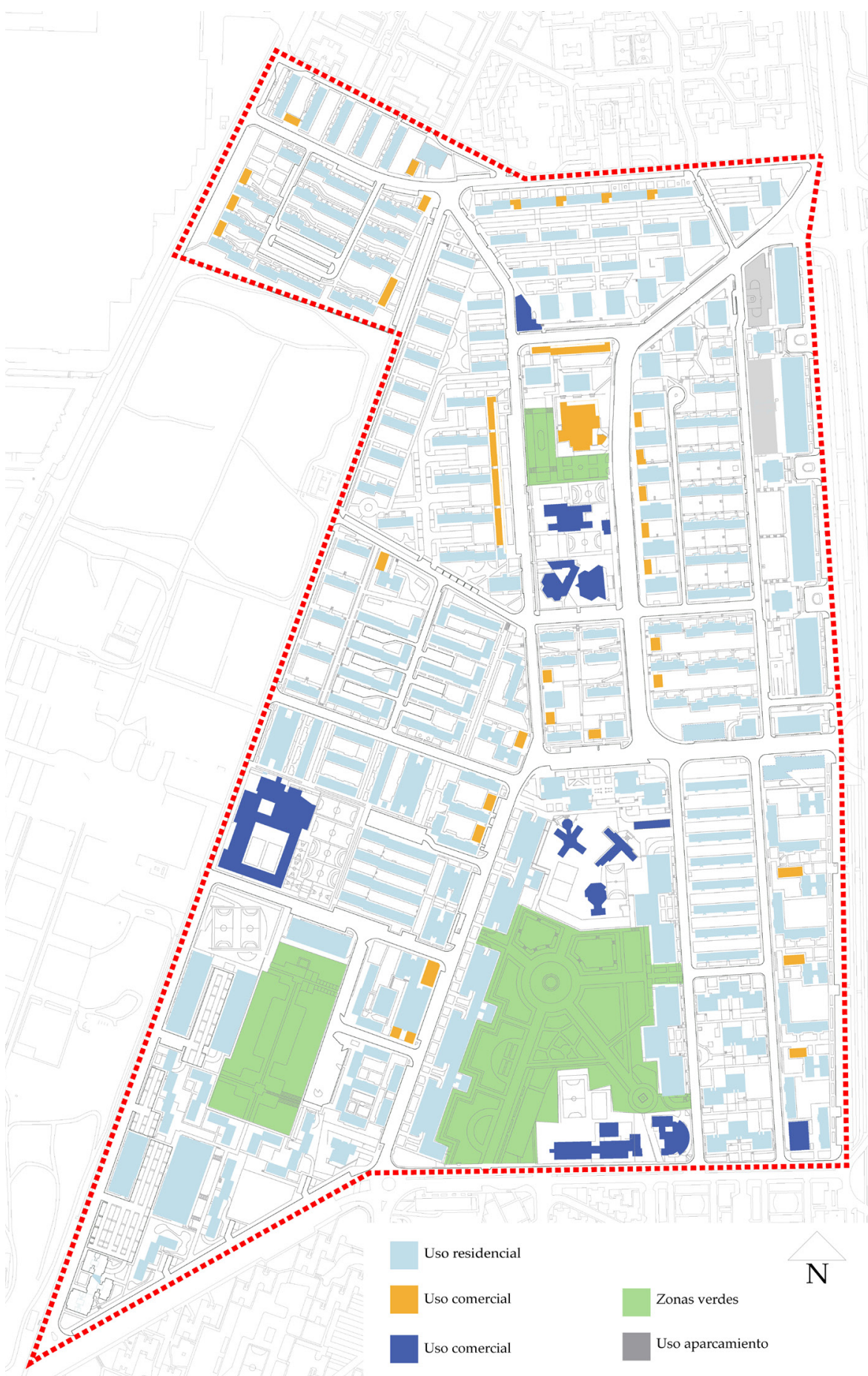

Distribución de usos 


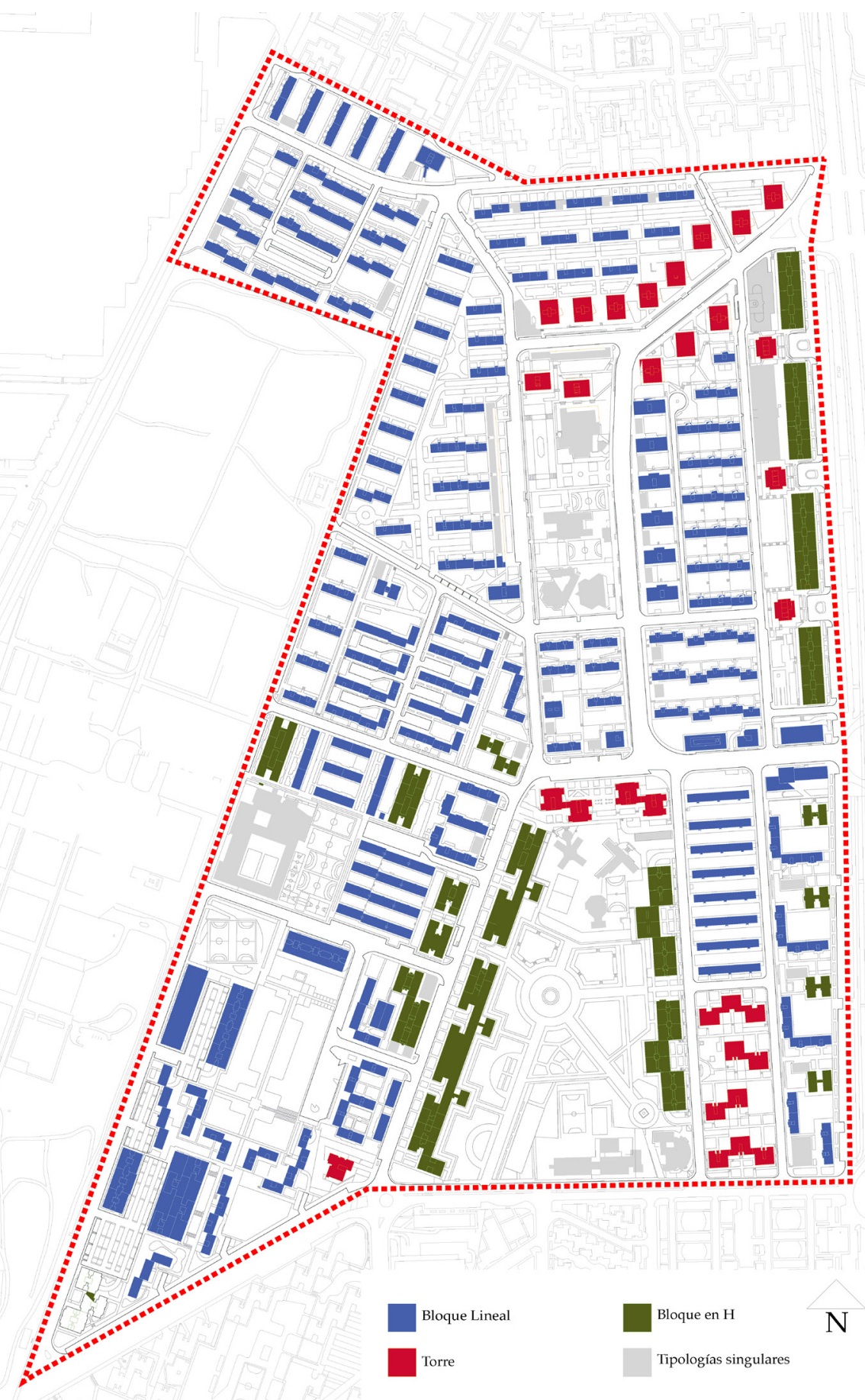


Dos constantes de su obra se reconocen en este barrio: la racionalidad, con la que vuelve su mirada a su trayectoria previa a la Guerra Civil, exilio y posterior destierro, y la importancia del espacio libre, que en la Ciudad de los Ángeles alcanza hasta el $80 \%$, con cuidados jardines aterrazados entre las edificaciones (Lasheras y Martín Moratalla 1987) ${ }^{6}$.La pureza volumétrica, la sinceridad constructiva, la ausencia de ornamento, de modo que el hueco se recorta limpiamente en el muro, la adecuada orientación e iluminación de las piezas, son preocupaciones coincidentes en otras de sus obras de este periodo de finalización de su vida profesional7, donde las referencias a sus emblemáticas Casa de las Flores (1930) y al proyecto de Nuevos Ministerios (1932) son evidentes, pero ahora resueltas con mayor libertad.

Estos valores patrimoniales del barrio están muy ligados a entenderlo como un espacio concebido para el desenvolvimiento de la vida cotidiana, convirtiendo al espacio físico en facilitador de las relaciones que constituyen el capital social del barrio. Fue concebido con una estructura fundamentada en un área central a partir del cual se organizan los polígonos que alojan fundamentalmente una tipología de bloques abiertos. Aunque el desarrollo del proyecto abarcó diferentes fases, es predominante el bloque abierto en diferentes configuraciones y orientaciones, constituyendo un entorno fácilmente reconocible de paralelepípedos puros y una gran generosidad de espacios libres. Las dotaciones y las principales zonas verdes del barrio se localizan en el área central, mediante una sucesión de lugares que facilitan la comprensión del espacio urbano y lo dotan de diversidad funcional y tipológica.

En el caso de Ciudad de los Ángeles supuso convertir a los trabajadores de las industrias adyacentes en propietarios de su vivienda, con la idea, entre otras, de mejorar las condiciones de habitabilidad de las familias ${ }^{8}$, con espacios urbanos saludables y de calidad.

Además de estas galerías, la continuidad de los bloques residenciales se consigue interponiendo entre ellos pequeños volúmenes comerciales, muchos de ellos ligados a la edificación con marquesinas de hormigón, dando muestras de la voluntad por conseguir una unidad del conjunto urbano y edificatorio propio de esta época.

\section{RECUPERACIÓN DEL PATRIMONIO Y APEGO AL LUGAR: EL PAPEL DE LA INVOLUCRACIÓN VECINAL}

Antes de valorar el tratamiento de los valores patrimoniales en la rehabilitación de Ciudad de los Ángeles, merece la pena detenerse en el concepto de "apego al lugar"9. La experiencia europea en regeneración urbana indica cómo la implicación vecinal en el proceso es un elemento central (Roberts y Sykes 2000; Kleinhans, Premius y Engbersen 2007; Premius 2008; Stouten
Además, otro de los valores que distingue a la Ciudad de los Ángeles, y la unen a las experiencias planificadoras de Zuazo, es el compromiso urbano de sus edificaciones, de 5 y 12 plantas, que se adaptan a la topografía, crean centralidad, con sus locales comerciales y equipamientos en la planta baja, abiertos a la vía principal, y producen una armónica disposición de sus manzanas paralelas, que es fruto de la agrupación de sus bloques abiertos, alternados con torres, enfatizadas en este caso sus cubiertas con potentes marquesinas.

$$
7
$$

El arquitecto bilbaíno Secundino Zuazo falleció el 12 de julio de 1971 en Madrid, después de una larga vida y completamente rehabilitado profesionalmente, respetado y admirado. Aunque apenas se menciona el proyecto de la Ciudad de los Ángeles, figurando en la relación de sus obras, sigue siendo imprescindible para el conocimiento de la figura del arquitecto el estudio de Lilia Maure (1987).

$$
8
$$

Es interesante para el entendimiento del papel de los industriales de Villaverde, la película dirigida por Besas en 1987 acerca del industrial Eduardo Barreiros (Barreiros, motor humano), donde se muestra su intervención en la Ciudad de los Ángeles.

\section{9}

En la literatura anglosajona suele aparecer como place-attachment. 
10

Con la preocupación sobre la recuperación de las periferias en Europa, algunos autores han retomado o continuado, según los casos, con la utilización de este concepto para, por un lado, poner de manifiesto la necesidad de institucionalizar la involucración de los vecinos en las dinámicas de transformación y mejora de sus barrios, y, por otro, comprender cómo la mejora de los espacios colectivos de los barrios (equipamientos y espacios públicos) son elementos catalizadores, generadores de ese capital social (Kearns 2004; Flint y Kearns 2006; Curley 2008; Svendsen 2010; Hudson 2012).

\section{1}

Entendemos que el capital social es el conjunto de beneficios y el modo de acceso a beneficios que tienen una serie de personas que forman parte de una red de relaciones basadas en la confianza, en las que se aplican unas normas que garantizan alguna forma de reciprocidad (Bourdieu 1980; Portes 1998; Edwards y Foley, 1999; Putnam 2000; Lin y Erikson 2008; González González 2017). Para Bourdieu, el concepto de capital social es algo más restringido: es el conjunto de recursos asociados a la posesión de una red de relaciones duraderas (Bourdieu 2000). Cuando Coleman (2000) introduce el concepto dentro del mundo académico e intelectual anglosajón, abre el camino a las aportaciones de Portes (1998), Lin (2001), Fine (2001), Putnam (2000), etc. que consideran el capital social inmerso de lleno en la esfera urbana. Putnam (2000) hace bascular e concepto hacia la confianza y la reciprocidad, lo que lleva a la aplicación del concepto en relaciones de sociabilidad más difusa, como son las vecinales.

\section{2}

El apego al lugar otorga una identidad colectiva reconocible (González González 2019). Curley (2010) muestra empíricamente cómo la pertenencia al lugar que conlleva este vínculo estimula las interacciones sociales que están en la base de la formación del capita social. La consciencia compartida de pertenecer a un lugar geográfico lleno de connotaciones predispone a construir vínculos solo
2010; González González y Stouten 2014). En la primera década del siglo XXI, Forrest y Kearns (2001), se preguntaban en su trabajo "Social cohesion, social capital and the neighbourhood", casi convertido hoy en un clásico, qué podía aportar el barrio como espacio de convivencia a la generación de capital social entre los vecinos y, de modo recíproco, qué es lo que el capital social de los vecinos aporta al mantenimiento de su espacio físico, en definitiva, a su rehabilitación ${ }^{10}$.

Además de señalar la importancia de este capital social, más operativo cuanto más institucionalmente organizado esté, los autores destacaban cómo "el apego al lugar" era un factor fruto de la convivencia en un mismo sitio identificable, y que formaba parte de una base de procesos informales sobre los que construir beneficios comunes e individuales, derivados de compartir una serie de relaciones de confianza y reciprocidad entre vecinos, y ser regidos por unas normas informales consensuadas. Estos beneficios y las relaciones que implican es lo que denominaríamos capital social ${ }^{11}$.

En continuidad de las propuestas de estos autores podemos decir que la implicación vecinal en un proceso de rehabilitación supone poner a actuar al capital social del barrio (González González 2019), lo que conlleva reforzar el denominado "apego al lugar", como reflejo de la construcción colectiva de un imaginario compartido ${ }^{12}$, que muchas veces, concuerda con los valores patrimoniales reconocidos y a preservar.

Creemos que en el caso de Ciudad de los Ángeles se da una doble implicación; por un lado, el "apego al lugar" involucra a los vecinos en la rehabilitación del barrio y, en segundo lugar, la rehabilitación, que respeta el imaginario colectivo, refuerza el sentimiento de "apego". Como conclusión: la estima de los valores patrimoniales del barrio se codifica, para los vecinos en términos de "apego al lugar, y, si este se refuerza, es que, de algún modo, se respetan dichos valores, se tienen en cuenta en la ecuación rehabilitadora. Por tanto, es un buen ejemplo de cómo el capital social del barrio es capaz de empujar el proceso de rehabilitación. En este caso, el capital social más institucionalizado se encuentra en la asociación de vecinos, ASVEYCO, y su papel de mediación entre las comunidades de propietarios y la administración local fue un elemento importante en el proceso de rehabilitación del barrio.

En Ciudad de los Ángeles, la asociación de vecinos tiene un rol complementario al del ente gestor ${ }^{13}$, ganando a lo largo del proceso un más intenso papel de representación de las comunidades implicadas en el área a rehabilitar ${ }^{14}$. Indudablemente, esta capacidad de interlocución de ASVEYCO se refleja en las propuestas de transformación física, condicionada por las características espaciales del barrio ya que el diseño urbano ha demostrado tener una clara presencia en la imagen colectiva y en la identificación de la población residente con los valores asociados a ella. De esta forma, el capital social habría 


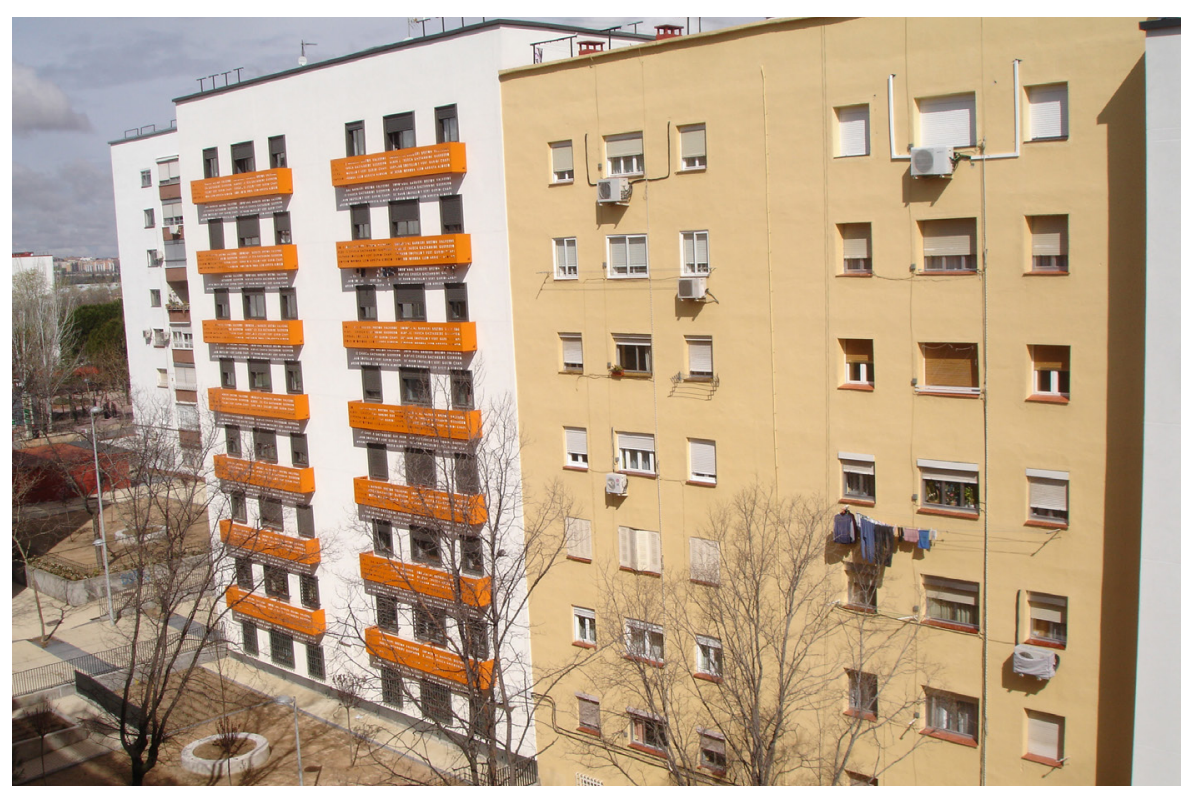

sido capaz de producir su propio espacio asimilando los valores del espacio concebido por el estudio Zuazo.

\section{Los valores patrimoniales en el proceso de rehabilitación}

En gran medida la rehabilitación de Ciudad de los Ángeles no se detuvo en los valores patrimoniales descritos hasta el momento, y no fueron considerados como objetivo dentro de la estrategia de rehabilitación adoptada por la EMVS ni por ASVEYCO (Córdoba Hernández et ál. 2020).

Pese a ello, la rehabilitación se definió en torno a unos parámetros que, a la larga, han podido influir sobre estos aspectos más de lo que hubiese podido pensarse. Cabe resaltar que en ningún momento se planteó la sustitución de la edificación y que, al contrario, se defendió su tratamiento unitario, aceptando la incorporación de elementos de color y composición de fachadas que permitiera una identificación de la población con su edificio sin que por ello se desvirtuara el sentido unitario del entorno ni la pureza volumétrica existente. Este volumen puro sí que ha sido parcialmente modificado con dos elementos que merecen su atención: las cajas de ascensores y la instalación de cubrevistas.

La instalación de ascensores en los bloques de cuatro o cinco plantas que carecían de él plantea la discusión sobre la necesidad de solventar la accesibilidad a costa de los valores arquitectónicos y urbanos de un determinado entorno o edificio. Como mencionamos, entre los objetivos para la rehabilitación de Ciudad de los Ángeles nunca se planteó esta disyuntiva y no se
A la izquierda, estado reformado del edificio de Pan y Toros 24-26 y abajo detalle de los cubrevistas de mismo edificio

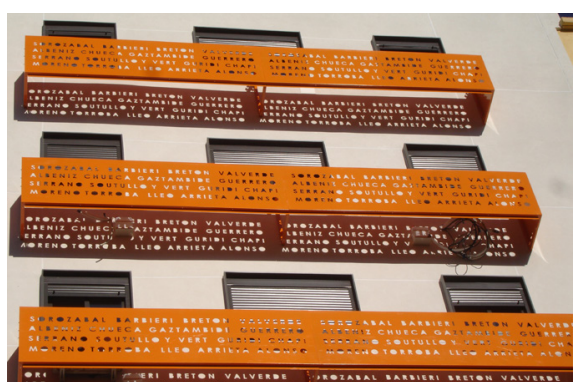

por compartir el lugar de residencia (González González 2019).

13

En el modelo habitual de gestión de las áreas de rehabilitación en Madrid, el ente gestor es la Empresa Municipal de Vivienda y Suelo (EMVS) del Ayuntamiento, que abre una Oficina de Rehabilitación en los propios barrios, y desde ella se tramitan las ayudas a las comunidades de propietarios, se ofrece información en general y se facilita apoyo técnico.

14

En el convenio firmado por el Ayuntamiento de Madrid y la Comunidad de Madrid para la declaración de Zona de Rehabilitación Integrada, se reconoce el papel de ASVEYCO, de representación vecinal. Esta mediación se inicia cuando problemas estructurales debidos a deficiencias de cimentación llevan a la asociación a convocar a 486 comunidades para pedir la declaración de ARI (Área de Rehabilitación Integral) para el barrio. La propuesta es aceptada por 248 comunidades, a las que se irán sumando más durante el proceso hasta alcanzar 411, lo que supone 7.996 viviendas. Lo significativo del caso es que el capital so- 
cial acumulado de ASVEYCO es capaz de iniciar el proceso gracias a su predicamento en el barrio, fundamentado en su defensa de los intereses de los vecinos, además de su labor como centro cultural y social local. También tiene importancia el mecanismo engrasado de relación con las comunidades en la gestión diaria. Se demostró muy útil que las relaciones de cobro de recibos habituales se hicieran directamente con las comunidades y no con los vecinos que viven en ellas. Esto supone un sistema ya maduro de toma de decisiones dentro y de interlocución hacia fuera, hacia la asociación. Otros aspectos de esta mediación es la gestión de la selección de una asistencia técnica para la petición de subvenciones y de empresas constructoras que trabajen para cada comunidad. La empresa gestora que resulta ganadora del concurso impulsado desde la asociación se encargará del desarrollo de los proyectos técnicos y de la dirección de obras de aquellas comunidades que así lo decidieran. Aunque en un principio se pretendía que este mecanismo rehabilitara los edificios de todas las comunidades, finalmente cada comunidad optó por el mecanismo de gestión que estimó adecuado, perdiendo de alguna manera la intensidad del elemento unificador de la gestión. En definitiva, la experiencia de Ciudad de los Ángeles nos remite a la gestión vecinal de un proceso complejo, como es el de la rehabilitación de un barrio, con una labor de mediación entre vecinos y administración. Esta labor no hubiera sido posible si no se contara con un músculo organizativo amplio y con medios materiales suficientes. También influye la inserción en la vida cotidiana del barrio gracias a su labor como centro social que ofrece servicios complementarios a los del ayuntamiento y otras instituciones (González González 2019).

Estado previo del edificio de La Corte del Faraón 25 (arriba izquierda) y del estado reformado (arriba derecha). Estado previo del edificio de EI Manojo de Rosas 2 (abajo izquierda) y del estado reformado (abajo derecha) generó ninguna duda respecto de las necesidades demandadas por el movimiento vecinal y la población residente. Sí se buscó, en cambio, la mayor coherencia y unidad posible (Morcillo Álvarez, Arjona Martín y Martín Acosta 2016), estableciendo áreas de movimientos en cada edificio, tras un minucioso estudio de cada tipología y modelo existente. Este trabajo fue incorporado al Plan Especial para la Instalación de Ascensores que fue aprobado en 2008 por el Ayuntamiento de Madrid.

Consideración diferente puede mostrarse en la decisión de instalar cubrevistas que organizaran la fachada de tal forma que ocultaran los aparatos de
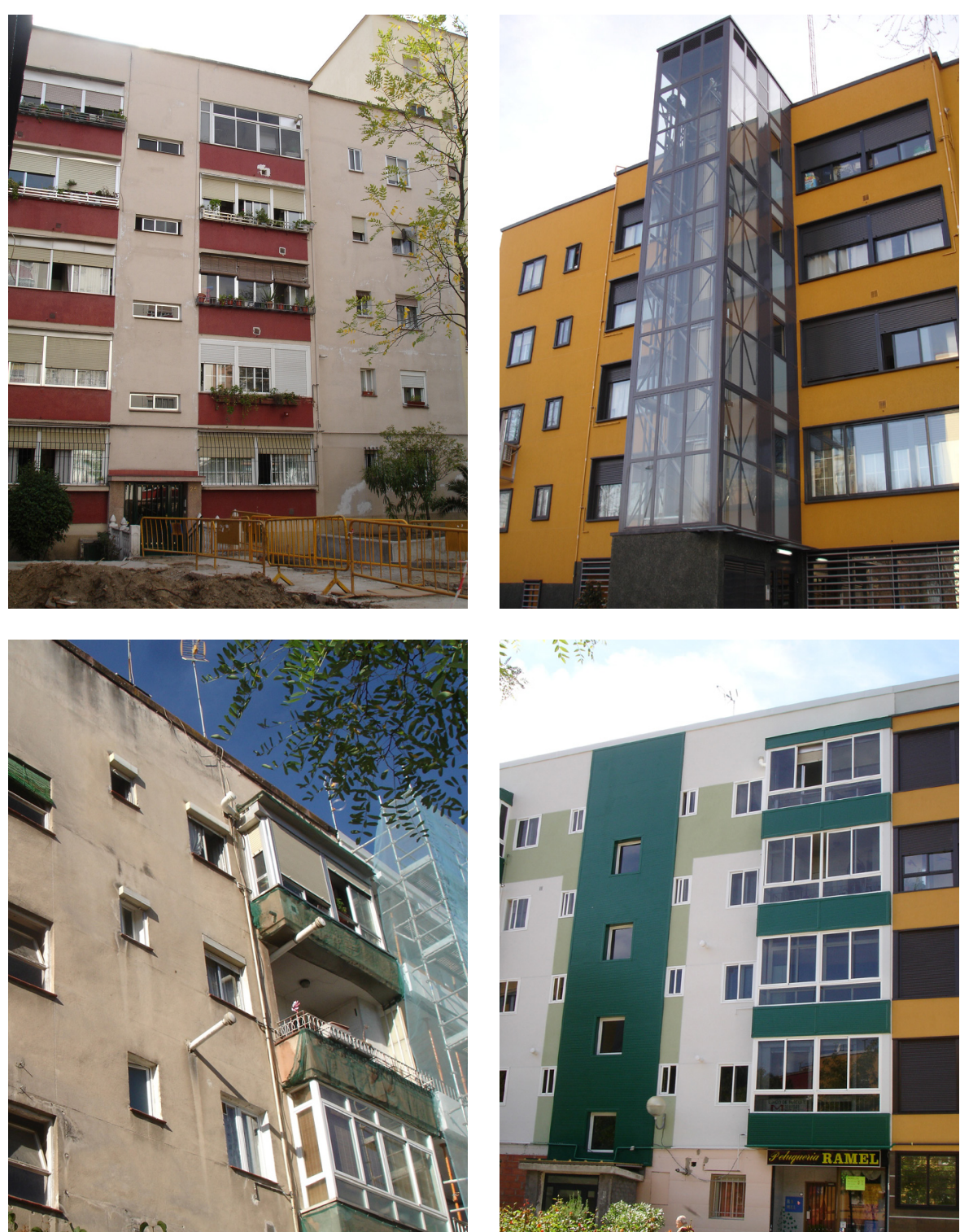
aire acondicionado, los tendederos y otros elementos fijados a las fachadas. La pérdida de la pureza compositiva puede ser discutible pero el tratamiento unitario, la voluntad repetitiva y el uso de este elemento como instrumento del nuevo lenguaje arquitectónico pueden estar en sintonía con los planteamientos industriales, repetitivos y seriados señalados anteriormente. Los cubrevistas dan un orden a la fachada que había sido perdido por la aparición de numerosos elementos ajenos al edificio.

Sin embargo, gran parte de la actuación se fundamentó en resolver los problemas derivados de la falta de conservación y mantenimiento del espacio
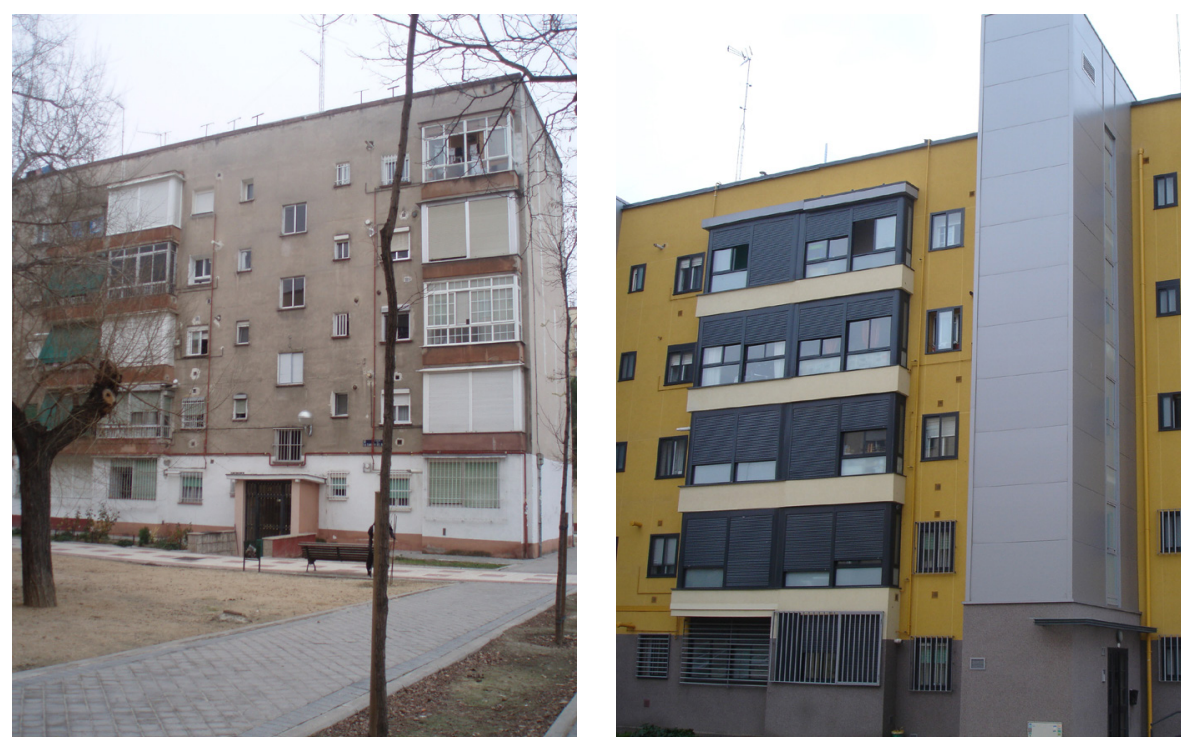

Estado previo del edificio de La Canción del Olvido 21 (arriba izquierda) y del estado reformado (arriba derecha). Estado previo del edificio de El Manojo de rosas 4 (abajo izquierda) y del estado reformado (abajo derecha)
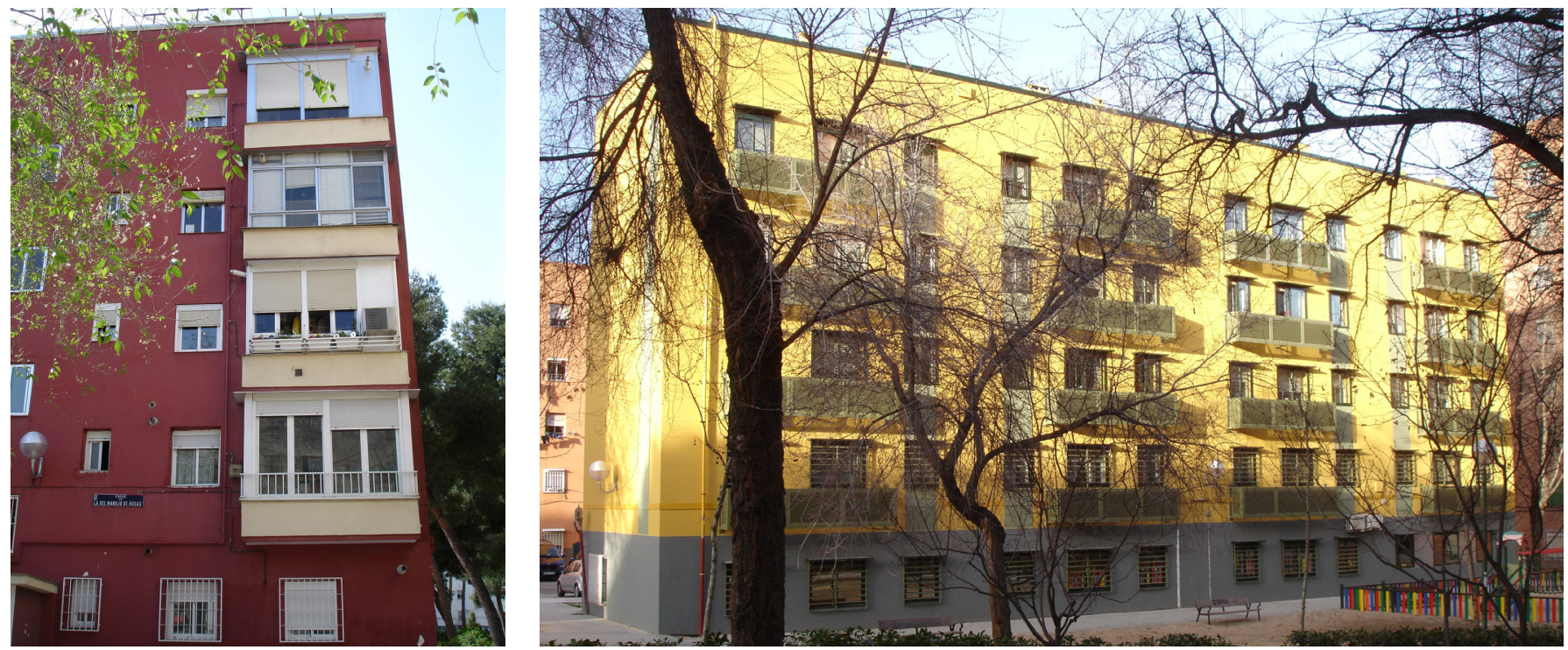
Taller de participación vecinal para la toma de decisiones
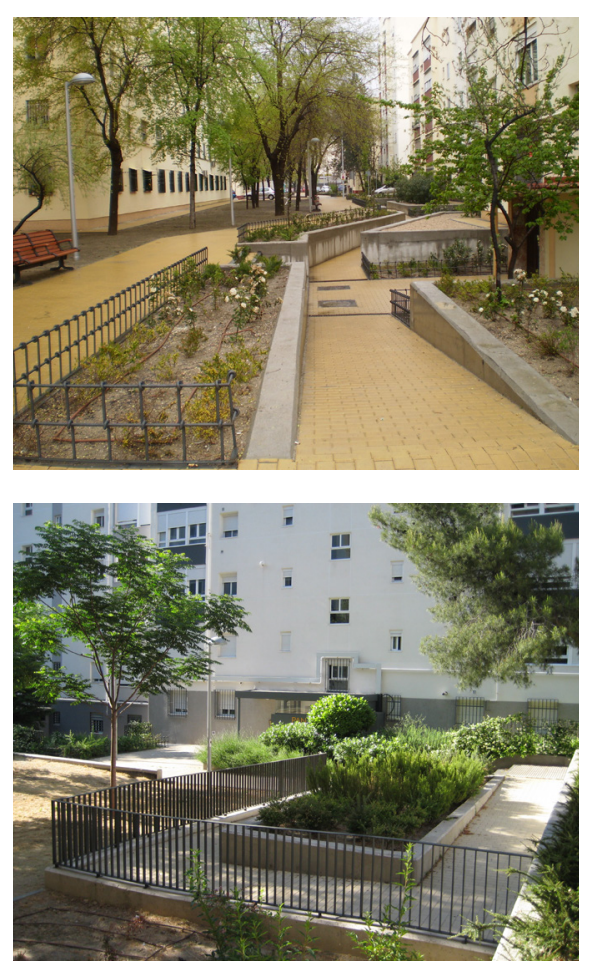

Rehabilitación urbana y solución de la accesibilidad a portales y terrazas. Calles Manojo de Rosas y Pan y Toros

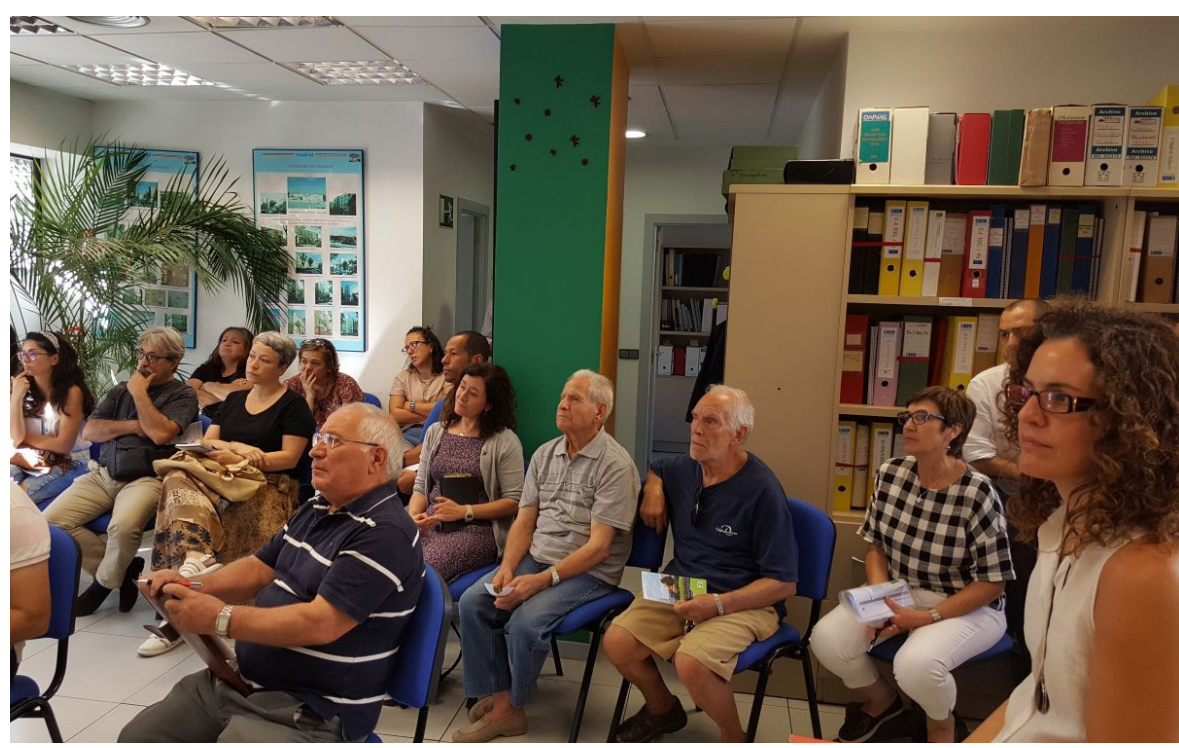

libre y que habían hecho que gran parte de ellos fueran conservados por la población. Esto provocó en el barrio un sentimiento de apropiación del espacio público y la identificación de la población con el lugar, secuencia esencial del "apego al lugar" del que ya hemos hablado.

Este hecho fue determinante en la participación de ASVEYCO y, en general, de la población residente, en el proceso de redacción de los proyectos de urbanización, no solo formando parte de la toma de decisiones en cuanto a las fases a desarrollar, sino incluso en el diseño, participando de la toma de decisiones de la elección de la vegetación, la localización del mobiliario y los usos del espacio. De esta manera, la rehabilitación ha dado un protagonismo especial al pasado de la población, sus costumbres y sentido de apropiación del espacio.

Es así como podemos entender que se ha llevado a cabo una protección ambiental del barrio derivada de la labor de vigilancia de los proyectos y la respuesta de los vecinos como parte de aceptación del resultado.

La rehabilitación del espacio público se fundamentó en resolver gran parte de los problemas de accesibilidad existentes, incorporar nuevas zonas vegetales y reducir, en general, el impacto del vehículo en un barrio de indudable calidad ambiental y que estaba absorbido por el exceso y descontrol de los aparcamientos. La unidad de actuación, a través del reconocible muro de hormigón y del adoquinado dorado, ha dotado al barrio de una imagen unitaria que ha solventado la gran fragmentación existente dado por años de descoordinación y de falta de una adecuada conservación de los generosos espacios libres. 


\section{CONCLUSIONES PARA FUTURAS INTERVENCIONES EN BARRIOS CON VALORES PATRIMONIALES CONTEMPORÁNEOS}

La rehabilitación de Ciudad de los Ángeles no ha considerado los aspectos patrimoniales como objetivos prioritarios a los que atender de modo explícito. Sin embargo, lo que podríamos considerar la "protección ambiental" del barrio se mantiene debido a la existencia de un consenso sobre "lo razonable" entre todos los agentes implicados.

Así, en lo que se refiere a los proyectos de rehabilitación de los arquitectos participantes y su control desde la administración, las propuestas intentan incorporar elementos de mejora de la habitabilidad como ascensores, tratamiento de huecos, reorganización de las instalaciones vistas en fachada, etc. Para estas rehabilitaciones se establecen unos criterios de intervención desde la gestión municipal que define el marco de esta "protección ambiental", de tal manera que se ha buscado la coherencia en la unidad del tratamiento de la fachada, incluso cuando se incorporan los ascensores, al delimitar áreas de movimiento determinadas para cada tipología.

Por otro lado, los vecinos sienten que siguen viviendo en su barrio y los cambios no son rechazados porque no transgreden su imaginario colectivo sobre lo que el barrio era, es decir, sobre sus valores patrimoniales. El "apego al lugar" sigue intacto, si no reforzado. Las innovaciones formales y espaciales no se entienden como ataques, sino como elementos de mejora, nunca como atentados contra la identidad colectiva de su barrio.

En el tratamiento de los espacios públicos queda patente esa intención de protección ambiental al haberse mantenido las trazas originales, recuperando las calles estructurantes, algo que permite reforzar la legibilidad del espacio original y reforzar los usos comerciales y los equipamientos que forman los espacios centrales del barrio. La mejora de la estancialidad y la movilidad peatonal en el barrio ha permitido reforzar los usos del espacio público contribuyendo a la producción de la identidad colectiva y el apego al lugar ${ }^{15}$.

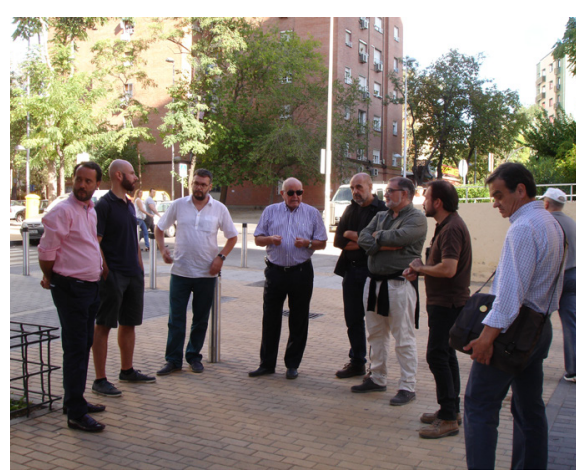

Taller técnico con vecinos. Visita a las rehabilitaciones ya ejecutadas

15

Sin menoscabo de los aciertos encontrados en el proceso de rehabilitación, sí se pueden identificar algunas deficiencias o errores que son útiles para reflexionar sobre futuras actuaciones en barrios con estas características. Así, a pesar de estos criterios aportados desde la gestión pública y que se negociaban con los arquitectos que acometían la rehabilitación, al no ser normativos, se detectan algunas malas prácticas puntuales referidas fundamentalmente a la falta de coordinación en el tratamiento de fachadas entre dos portales de un mismo bloque, el uso de materiales inadecuados y la sustitución y supresión de elementos valiosos del espacio público como las marquesinas de hormigón y las pérgolas que daban unidad compositiva entre los bloques lineales residenciales y los bloques comerciales. 


\section{BIBLIOGRAFÍA}

- Bourdieu P. (1980) Le capital social: notes provisoires. Actes de la Recherche en Sciences Sociales, vol. 31, pp. 2-3

- Coleman, J.S. (2000) Social capital in the Creation of Human Capital. En: Lesser, Eric L. (ed.) Knowledge and Social Capital. Foundations and Applications. Boston: Butterworth Heineman

- Córdoba Hernández, R., Carmona Mateos, F., Morcillo Álvarez, D., Román López, E., y Sánchez-Guevara, C. (2020) Área de Regeneración Urbana. Ciudad de los Ángeles. Ciudad y Territorio: Estudios territoriales, vol. LII, n. ${ }^{\circ}$ 204, pp. 349-356. Disponible en: https://doi org/10.37230/СуTET.2020.204.11 [Consulta: 11/06/2021]

- Curley, A.M. (2010) Relocating the poor: social capital and neighborhood resources. Journal of urban affairs, n. ${ }^{\circ} 32$, pp. 79-103. Disponible en: https://doi.org/10.1111/ j.1467-9906.2009.00475.x [Consulta: 11/06/2021]

- Edwards, B. y Foley, M. (1999) Is it time to disinvest in social capital? Journal of Public Policy, vol. 19, n. ${ }^{\circ} 2$, pp. 141-173

- Elvira Peña, J. (2003) Secundino Zuazo y la ciudad de Los Ángeles. En: Sambricio, C. (coord.) Un siglo de vivienda social: 1903-2003 [Catálogo de la exposición organizada por el Ministerio de Fomento, el Ayuntamiento de Madrid-EMV y el Consejo Económico y Social (CES)] Madrid: Nerea, vol. 2, tomo 2, pp. 92-93

- Fine, B. (2001) Social capital versus social theory: political economy and social science at the turn of the millennium. London: Routledge

- Flint, J. y Kearns, A. (2006) Housing, neighbourhood renewal and social capital: the case of registered social landlords in Scotland. European Journal of Housing Policy, vol. 6, n. $^{\circ} 1$, pp. $31-54$

- Forrest, R. y Kearns, A. (2001) Social cohesion, social capital and the neighbourhood. Urban Studies, vol. 38, n. $^{\circ}$ 12, pp. 2125-2143

- González González, F.J. y Stouten, P. (2014) Purper en Amber, Poptahof, Delft. Ciudad y Territorio, Estudios Territoriales, vol. XLVI, n. ${ }^{\circ} 179$, pp. 175-181. Disponible en: https://recyt.fecyt.es/index.php/CyTET/article/view/76298 [Consulta: 10/06/2021]

- González González, F.J. (2017) Capital social y diversidad articulada en el espacio público. Influencia de las condiciones morfológicas y funcionales de las plazas en el refuerzo de la cohesión social en barrios de Madrid con procesos de regeneración urbana. Tesis doctoral inédita. Universidad Europea de Madrid. Disponible en: http://hdl. handle.net/11268/6982 [Consulta: 11/06/2021]

- González González, F.J. (2019) Confianza y capital social en la regeneración urbana de barrios y conjuntos de viviendas. En: López-Mesa, B. (coord.) Nuevos enfoques en la rehabilitación energética de la vivienda hacia la convergencia europea: La vivienda social en Zaragoza, 1939-1979, vol. 2. Zaragoza: Prensas de la Universidad de Zaragoza, pp. 281-294 (Cátedra Zaragoza Vivienda). Disponible en: https://catedrazaragozavivienda. files.wordpress.com/2019/01/3.4-generaci\%C3\%B3nconfianza.pdf [Consulta: 11/06/2021]

- Gutiérrez Marcos, J. y Da Rocha, O. (2007) Villaverde Usera. En: Lasso de la Vega, M. (coord.) Arquitectura de Madrid. Periferia. Madrid: Fundación COAM, vol. 3, pp. 567-568

- Hudson, A. (2012) Does residential access to public space contribute to higher levels of social capital? A case study in Southern Oregon. SOU McNair Scholars Journal, vol. 8

- Kearns, A. (2004) Social capital, regeneration and urban policy. Centre for Neighbourhood-CNR Paper 15

- Kleinhans, R., Priemus, H. y Engbersen, G. (2007) Understanding social capital in recently restructured urban neighbourhoods: Two case studies in Rotterdam. Urban Studies, vol. 44, n. ${ }^{\circ}$ 5-6, pp. 1069-1091

- Lasheras, C. y Martín Moratalla, M.A. (1987) Ciudad de los Ángeles. En: Madrid proyecto Madrid 19831987. Madrid: Área de Urbanismo e Infraestructuras, Ayuntamiento de Madrid

- Lasso de la Vega, M. (2008) El Instituto Nacional de la Vivienda de Federico Mayo y José Fonseca. En: Sambricio, C. y Sánchez Lampreave, R. (ed.) 100 años de intervención pública en la vivienda y la ciudad. Madrid: Asociación Española de Promotores Públicos de Vivienda y Suelo AVS, pp. 43-72

- Ley 84/1961, de 23 de diciembre, sobre Plan Nacional de la Vivienda para el periodo 1961-1976. Boletín Oficial del Estado, n. ${ }^{\circ} 310$, de 28 de diciembre de 1961. Disponible en: https://www.boe.es/buscar/doc. php?id=BOE-A-1961-23794 [Consulta: 10/06/2021]

- Lin, N. (2001) Social capital. A theory of social structure and action. Cambridge: Cambridge University Press (Structural analysis in the social sciences; 19)

- Maure Rubio, L. (1987) Secundino Zuazo, arquitecto. Madrid: Colegio Oficial de Arquitectos de Madrid

- Morcillo Álvarez, D., Arjona Martín, C. y Martín Acosta, N. (2016) Recuperando la periferia. La rehabilitación energética de Ciudad de los Ángeles en Madrid. Ciudad y territorio: Estudios territoriales, vol. XLVIII, n. ${ }^{\circ}$ 187, pp. 57-76. Disponible en: https://apps.fomento.gob.es/CVP/ handlers/pdfhandler.ashx?idpub=BP1015 [Consulta: 11/06/ 2021] 
- Portes, A. (1998) Social Capital: Its origins and application in Modern Soiciology. Annual review of Sociology, n. ${ }^{0} 24$, pp. 1-24

- Premius, H. (2008) Vivienda Social y renovación urbana en Holanda. En: Leal, J. (de.) Rehabilitación de viviendas y renovación urbana en las grandes ciudades europeas. Madrid: Ayuntamiento de Madrid

- Putnam, R.D. (2000) Bowling Alone: The Collapse and Revival of American Community. New York: Simon \& Schuster

- Río Lafuente, I. (Del) (1984) Industria y residencia en Villaverde: génesis de un paisaje urbano en la periferia de Madrid. Madrid: Universidad Complutense

- Ripoll Gómez, C. y Ripoll Tolosana, V. (2014). La arquitectura del ocio en Manuel Muñoz Monasterio. En: Couceiro Núñez, T. (coord.) Actas del I Congreso Pioneros de la Arquitectura Moderna Española: Vigencia de su pensamiento y obra. Madrid: Fundación Alejandro de la Sota, pp. 813-822

- Roberts, P. y Sykes, H. (ed.) (2000) Urban Regeneration. A Handbook. London: Sage

- Roch Peña, F. (2008) La deriva patológica del espacio social en el modelo inmobiliario neoliberal madrileño. $X$ Coloquio Internacional de Geocrítica. Barcelona: Univer sidad de Barcelona

- Sambricio, C. (1999) La vivienda en Madrid, de 1939 al Plan de Urgencia Social, en 1959. En: La vivienda en Madrid en la década de los cincuenta: el Plan de Urgencia Social. Madrid: Electa, pp. 13-84

- Stouten, P.L. (2010) Changing contexts in urban regeneration: 30 years of modernisation in Rotterdam. Amsterdam: Techne Press

- Svendsen, G.L.H. (2010) Socio-spatial Planning in the Creation of Bridging Social Capital: The Importance of Multifunctional Centers for Intergroup Networks and Integration. International Journal of Social Inquiry, vol. 3, n. ${ }^{\circ} 2$, pp. $45-73$ 